\title{
Introduction to research topic - Brain connectivity analysis: investigating brain disorders. Part 2: original research articles
}

\author{
Silvina G. Horovitz ${ }^{1 *}$ and Barry Horwitz ${ }^{2}$ \\ Human Motor Control Section, National Institute of Neurological Disorders and Stroke, National Institutes of Health, Bethesda, MD, USA \\ 2 Brain Imaging and Modeling Section, National Institute on Deafness and Other Communication Disorders, National Institutes of Health, Bethesda, MD, USA \\ *Correspondence: horovits@mail.nih.gov
}

Although the review articles discussed in part 1 (Introduction to research topic - Brain connectivity analysis: investigating brain disorders. Part 1: the review articles) give a nice overview of the scope of brain connectivity analysis as applied to brain disorders, the original research papers included in this collection enable the reader to see how one actually goes about employing such analyses on real data sets. In these articles we see the specific steps that are necessary to perform each particular connectivity analysis. We believe that these articles will be especially useful as guides for other researchers attempting brain connectivity analyses.

Several articles focus on normal brain function, but these papers are included because the subject groups that are investigated experience changing patterns of brain connectivity, and as such, share some of the conceptual difficulties that occur when studying brain disorders. Karunanayaka et al. (2011) evaluate the developmental trajectories of fMRI effective connectivity using linear structural equation modeling (SEM) during the verb-generate task in a large group of children whose ages ranged between 5 and 18 years. Allen et al. (2011) present an independent component analysis (ICA) of the effects of healthy aging (12-71 years) and gender on resting state fMRI networks. As with the previous paper, the study population is quite large (over 600 subjects). Both studies provide results that can act as baselines for future investigations. A much smaller-scale study by Beauchamp et al. (2010) employs fMRI-SEM to examine the interaction of sensory systems (visual and tactile) during multisensory integration. This is an important issue, especially in aged populations where deterioration of peripheral sensory systems is common.

Language processing is the topic addressed by three of the original research connectivity articles. Turken and Dronkers (2011) used both DTI tractography and resting state fMRI functional connectivity to investigate in healthy subjects the structural and functional connectivity of white matter pathways between left hemisphere brain regions critical for language comprehension. These critical regions were identified in an earlier voxel-based lesion-symptom mapping analysis of data from aphasic patients (Dronkers et al., 2004). Reading is the issue examined in the other two articles. Frye et al. (2010) compared young adult normal and dyslexic readers. They used Granger causality applied to MEG data to investigate the relation between effective connectivity between left inferior frontal gyrus and other brain areas and phonological decoding. The work of Davis et al. (2010) is interesting in that it shows how neuroimaging may impact education. They present DTI data analyzed using probabilistic tractography that were acquired from first-grade children who differed in their levels of responsiveness to reading instruction.
One of the most challenging aspects of studying the brain is its amazing adaptive capabilities. It often reorganizes itself in response to occurrences in the external world; that is why we are able to remember past events, why we are able to learn new skills, and why we send our children to school. But, the brain also can reorganize to changes within the brain itself, including disease. Thus, an important set of issues for understanding brain disorders is to determine which changes are compensatory and which are maladaptive. Three articles presented here investigate connectivity changes accompanying reorganization. Turner et al. (2011) report on an fMRI study of traumatic brain injury (TBI) patients. By combining brain-behavior and functional connectivity analyses, they sought to determine whether the compensatory brain changes they found in the patients represented functional reorganization (novel brain region recruitment) or altered functional engagement (differential recruitment of similar brain regions between patients and controls based on task demands). The other two articles focus on developmental changes in the visual system following loss of vision. The paper by Sani et al. (2010) used fMRI to investigate motion processing in sighted and blind (loss of vision from birth or before 2 years of age) adults. They compared the differences between the two groups in functional connectivity of the human middle temporal complex (hMT+), parts of which are activated in sighted subjects by visual motion and parts of which can be activated by non-visual motion. The third paper in this group, by Bock et al. (2010), is unique in that its study population consists of ferrets, thus allowing one to compare neuroimaging results with detailed histopathological data. The question they addressed relates to understanding the neural basis of the changes observed in DTI data following early bilateral enucleation of the eyes.

The remaining articles present connectivity analyses of data from patients with specific neurological and psychiatric disorders. Amyotrophic lateral sclerosis (ALS), a progressive neurodegerative disorder affecting motor neurons, is the topic of the article by Jelsone-Swain et al. (2010). They studied this disorder using resting state functional connectivity analysis, focusing on early stage ALS and directing their analysis to the connectivity changes of motor cortex. Another neurodegenerative disorder, Parkinson's disease (PD), was addressed by Kwak et al. (2010). They also employed resting state fMRI functional connectivity analysis, applying it to data acquired from PD patients as well as healthy controls. An interesting feature of this paper was the fact that the PD patients were studied in both the ON and OFF L-DOPA medication states (a drug used to treat the symptoms of PD). The psychiatric disorders examined by the remaining papers were depression and social phobia. Resting state fMRI connectivity analysis of depression was covered in the article by Veer et al. (2010), who applied ICA to their 
resting state fMRI data and identified thirteen relevant networks. In the paper by Danti et al. (2010), fMRI data obtained during a face perception task were analyzed using functional connectivity analysis in patients with social phobia and in control subjects, with the goal of examining communication between sensory and emotional processing brain areas.

\section{ACKNOWLEDGMENTS}

This work was supported by the Intramural Research Programs of NINDS (Silvina G. Horovitz) and NIDCD (Barry Horwitz).

\section{REFERENCES}

Allen, E. A., Erhardt, E. B., Damaraju, E., Gruner, W., Segall, J. M., Silva, R. F., Havlicek, M., Rachakonda, S., Fries, J., Kalyanam, R., Michael, A. M., Caprihan, A., Turner, J. A., Eichele, T., Adelsheim, S., Bryan, A. D., Bustillo, J., Clark, V. P., Feldstein Ewing, S. W., Filbey, F., Ford, C. C., Hutchison, K., Jung, R. E., Kiehl, K. A., Kodituwakku, P., Komesu, Y. M., Mayer, A. R., Pearlson, G. D., Phillips, J. P., Sadek, J. R., Stevens, M., Teuscher, U., Thoma, R. J., and Calhoun, V. D. (2011). A baseline for the multivariate comparison of resting-state networks. Front. Syst. Neurosci. 5:2. doi: 10.3389/ fnsys.2011.00002

Beauchamp, M. S., Pasalar, S., and Ro, T. (2010). Neural substrates of reliability-weighted visual-tactile multisensory integration. Front. Syst. Neurosci. 4:25. doi: 10.3389/ fnsys. 2010.00025

Bock, A. S., Olavarria, J. F., Leigland, L. A., Taber, E. N., Jespersen, S. N., and Kroenke, C. D. (2010). Diffusion tensor imaging detects early cerebral cortex abnormalities in neuronal architecture induced by bilateral neonatal enucleation: an experimental model in the ferret. Front. Syst. Neurosci. 4:149. doi: 10.3389/fnsys.2010.00149

Danti, S., Ricciardi, E., Gentili, C., Gobbini, M. I., Pietrini, P., and Guazzelli, M. (2010). Is social phobia a "mis-communication" disorder? Brain functional connectivity during face perception differs between patients with social phobia and healthy control subjects. Front. Syst. Neurosci. 4:152. doi: 10.3389/fnsys.2010.00152

Davis, N., Fan, Q., Compton, D. L., Fuchs, D., Fuchs, L. S., Cutting, L. E., Gore, J. C., and Anderson, A.W. (2010). Influences of neural pathway integrity on children's response to reading instruction. Front. Syst. Neurosci. 4:150. doi: 10.3389/fnsys.2010.00150

Dronkers, N. F., Wilkins, D. P., Van Valin, R. D. Jr., Redfern, B. B., and Jaeger, J. J. (2004). Lesion analysis of the brain areas involved in language comprehension. Cognition 92, 145-177.
Frye, R. E., Wu, M. H., Liederman, J., and Fisher, J. M. (2010). Greater pre-stimulus effective connectivity from the left inferior frontal area to other areas is associated with better phonological decoding in dyslexic readers. Front. Syst. Neurosci. 4:156. doi: 10.3389/fnsys.2010.00156

Jelsone-Swain, L. M., Fling, B. W., Seidler, R. D., Hovatter, R., Gruis, K., and Welsh, R. C. (2010). Reduced interhemispheric functional connectivity in the motor cortex during rest in limb-onset amyotrophic lateral sclerosis. Front. Syst. Neurosci. 4:158. doi: 10.3389/fnsys.2010.00158

Karunanayaka, P., Schmithorst, V. J., Vannest, J., Szaflarski, J. P., Plante, E., and Holland, S. K. (2011). A linear structural equation model for covert verb generation based on independent component analysis of FMRI data from children and adolescents. Front. Syst. Neurosci. 5:29. doi: 10.3389/fnsys.2011.00029

Kwak, Y., Peltier, S., Bohnen, N. I., Muller, M. L., Dayalu, P., and Seidler, R. D. (2010) Altered resting state cortico-striatal connectivity in mild to moderate stage Parkinson's disease. Front. Syst. Neurosci. 4:143. doi: 10.3389/fnsys.2010.00143

Sani, L., Ricciardi, E., Gentili, C., Vanello, N., Haxby, J. V., and Pietrini, P. (2010). Effects of visual experience on the human MT+ functional connectivity networks: an fMRI study of motion perception in sighted and congenitally blind individuals. Front. Syst. Neurosci. 4:159. doi: 10.3389/fnsys.2010.00159

Turken, A. U., and Dronkers, N. F. (2011). The neural architecture of the language comprehension network: converging evidence from lesion and connectivity analyses. Front. Syst. Neurosci. 5:1. doi: 10.3389/fnsys.2011.00001

Turner, G. R., McIntosh, A. R., and Levine, B. (2011). Prefrontal compensatory engagement in TBI is due to altered functional engagement of existing networks and not functional reorganization. Front. Syst. Neurosci. 5:9. doi: 10.3389/ fnsys.2011.00009

Veer, I. M., Beckmann, C. F., van Tol, M. J., Ferrarini, L., Milles, J., Veltman, D. J., Aleman, A., van Buchem, M. A., van der Wee, N. J., and Rombouts, S. A. (2010). Whole brain resting-state analysis reveals decreased functional connectivity in major depression. Front. Syst. Neurosci. 4:41. doi: 10.3389/fnsys.2010.00041

Received: 25 January 2012; accepted: 27 January 2012; published online: 13 February 2012. Citation: Horovitz SG and Horwitz B (2012) Introduction to research topic - Brain connectivity analysis: investigating brain disorders. Part 2: original research articles. Front. Syst. Neurosci. 6:4. doi: 10.3389/fnsys.2012.00004

Copyright $(5) 2012$ Horovitz and Horwitz. This is an open-access article distributed under the terms of the Creative Commons Attribution Non Commercial License, which permits non-commercial use, distribution, and reproduction in other forums, provided the original authors and source are credited. 